\title{
Role of 16S Ribosomal RNA in Etiological Diagnosis of Pyogenic Liver Abscess
}

\author{
Navneet Kaur ${ }^{1}$, Ashwini Agarwal², Guduru Gopal Rao ${ }^{3}$ \\ Keywords: $16 \mathrm{~S}$ rRNA, Diagnosis, Liver abscess. \\ Journal of Gastrointestinal Infections (2021): 10.5005/jp-journals-10068-3045
}

Liver abscess is an uncommon but life-threatening condition seen in all parts of the world with mortality rates ranging from 2.5 to 26.4\%. 'Liver abscesses are most commonly caused by either bacteria (pyogenic) or Entamoeba histolytica (amebic) infection. The incidence of liver abscesses varies between different parts of the world as also in the relative proportion of pyogenic and amebic abscesses. In India, amebic abscesses are more frequent than pyogenic abscesses whereas in the Western countries, bacterial abscesses are by far commoner except in patients who give history for travel or past residence in countries where $E$. histolytica is endemic. ${ }^{2}$

Advances in imaging, less invasive interventive procedures for source control, and antibiotic treatment of pyogenic liver abscesses have improved the outcomes and reduced the mortality of this condition. However, in making an etiological diagnosis, limitations of traditional culture methods are being increasingly recognized. Many studies on the etiology of pyogenic liver abscesses have described that the traditional culture methods fail to detect the causal bacteria in up to $30 \%$ of cases or may not fully detect all the bacteria causing the abscesses. ${ }^{1}$ The failure to culture organisms could be due to the inherent lack of sensitivity of culture methods, slow growth, fastidious growth requirements, or particular incubation needs. Furthermore, many patients with suspected liver abscess are treated with empirical antibiotics, which may significantly reduce the ability to successfully culture the causative organisms.

In the recent decades, molecular methods such as polymerase chain reaction (PCR) have transformed the etiological diagnosis of various infections and indeed have replaced traditional culturebased diagnosis. However, PCR-based methods are limited by the requirement of specific primers against specific targets. It follows that PCR-based methods cannot detect the etiology of infections where the range of organisms is diverse and the necessary primers are not included in the test. New molecular methods have emerged to overcome this important limitation of PCR.

One such method is the sequencing of $16 \mathrm{~S}$ ribosomal RNA (16S rRNA) in the pus aspirated from the liver abscess. The bacterial ribosome (70S ribosome) structurally consists of $30 \mathrm{~S}$ and $50 \mathrm{~S}$ subunits. The $30 \mathrm{~S}$ subunit is composed of mainly $16 \mathrm{~S}$ rRNA whereas $50 \mathrm{~S}$ subunit contains $5 \mathrm{~S}$ and $23 \mathrm{~S}$ rRNA. Importantly, $16 \mathrm{~S}$ rRNA is evolutionarily conserved and found in all the bacteria. Detection and identification of bacteria based on the $16 \mathrm{~S}$ rRNA sequence have become particularly useful because the gene encoding 16S rRNA has a "conserved" region that exists universally among bacteria and a hypervariable region that is specific for identifying bacteria at a species level. ${ }^{3}$ Analysis of entire $16 \mathrm{~S}$ gene sequences can even distinguish between strains of the same species of bacteria.
1,2Department of Microbiology, All India Institute of Medical Sciences, Rajkot, Gujarat, India

${ }^{3}$ Department of Microbiology, London North West University Healthcare NHS Trust, London, United Kingdom

Corresponding Author: Guduru Gopal Rao, Department of Microbiology, London North West University Healthcare NHS Trust, London, United Kingdom, Phone: +020 88692972, e-mail: ggopalrao@ nhs.net; gudurug@hotmail.com

How to cite this article: Kaur N, Agarwal A, Rao GG. Role of $16 \mathrm{~S}$ Ribosomal RNA in Etiological Diagnosis of Pyogenic Liver Abscess. J Gastrointest Infect 2021;11:1-2.

Source of support: Nil

Conflict of interest: None

Sequencing of $16 \mathrm{~S}$ rRNA gene involves extraction of DNA from clinical specimens and using it as a template for PCR to amplify a segment of about 500 or $1500 \mathrm{bp}$ of the 16S rRNA gene sequence. Bacteria can be identified by nucleotide sequence analysis of the PCR product followed by comparison of this sequence with known sequences stored in a database. ${ }^{3}$

Many recently published reports have employed 16S rRNA sequencing in the timely identification of unusual non-cultivable bacteria, which has helped in the early initiation of definitive antibiotic therapy in liver abscess. The identified bacteria using this technique comprise Eggerthella lenta, Aggregatibacter aphrophilus, Pannonibacter phragmitetus, Parvimonas micra, Streptococcus oralis, Fusobacterium spp, Bacteroides spp, Prevotella spp, Peptostreptococcus, Unassigned Enterobacteriaceae, etc. ${ }^{4-6}$

Clearly, 16S rRNA sequencing is proving to be useful in detection and identification of bacteria causing liver abscess, but it has important limitations. First, it does not provide antibiotic susceptibility data that have to be inferred from the identity of the organism. ${ }^{7}$ Second, low taxonomical resolution of the sequencing reads has been observed for some bacterial genus. Thirdly, for superior species identification, where interspecies hypervariable regions are similar as observed between Escherichia coli and Shigella spp. and between Streptococcus viridians; additional sequencing of other genes are needed. ${ }^{8}$ In addition, at a practical level, especially in resource-limited settings, establishing a laboratory for sequencing requires substantial funds and technically competent laboratory personnel. ${ }^{9}$

Provided resources and funding are available, $16 \mathrm{~S}$ rRNA has the potential to change our understanding of etiology of liver abscess and make an important contribution in patient's management. PCR 
based on 16S rRNA has also proven its diagnostic utility in etiological diagnosis of sepsis, infective endocarditis, and osteomyelitis particularly in culture-negative cases. Indeed, 16S rRNA promises to usher in a new era of diagnostic microbiology for a variety of infections.

\section{References}

1. Bivand JM, Nygaard RM, Kommedal Ø. Characterization of abscesses from liver, pancreas and kidney using deep sequencing of the $16 S$ rRNA gene. Diagn Microbiol Infect Dis 2021;99(3):115277. DOI: 10.1016/j.diagmicrobio.2020.115277.

2. Ali WM, Ali I, Rizvi SA, et al. Recent trends in the epidemiology of liver abscess in western region of Uttar Pradesh: a retrospective study. J Surg Anesth 2018;2(117):1-4. DOI: 10.35248/2684-1606.18.2.117.

3. Clarridge III JE. Impact of $16 \mathrm{~S}$ rRNA gene sequence analysis for identification of bacteria on clinical microbiology and infectious diseases. Clin Microbiol Rev 2004;17(4):840-862. DOI: 10.1128/ CMR.17.4.840-862.2004.
4. Okumura T, Soya K, Hihara Y, et al. The first known case of liver abscess caused by Aggregatibacter aphrophilus in Japan. Intern Med 2020;59(11):4220-4219. DOI: 10.2169/internalmedicine.4220-19.

5. Elias RM, Khoo SY, Pupaibool J, et al. Multiple pyogenic liver abscesses caused by Eggerthella lenta treated with ertapenem: a case report. Case Rep Med 2012;2012. DOI: 10.1155/2012/718130.

6. Wang $M$, Zhang X, Jiang T, et al. Liver abscess caused by Pannonibacter phragmitetus: case report and literature review. Front Med 2017;4: 48. DOI: $10.3389 / \mathrm{fmed} .2017 .00048$.

7. Neill L, Edwards F, Collin SM, et al. Clinical characteristics and treatment outcomes in a cohort of patients with pyogenic and amoebic liver abscess. BMC Infect Dis 2019;19(1):1-8. DOI: 10.1186/ s12879-019-4127-8.

8. Jenkins $\mathrm{C}$, Ling $\mathrm{CL}$, Ciesielczuk HL, et al. Detection and identification of bacteria in clinical samples by $16 \mathrm{~S}$ rRNA gene sequencing: comparison of two different approaches in clinical practice. J Med Microbiol 2012;61(4):483-488. DOI: 10.1099/jmm.0.030387-0.

9. Khim G, Em S, Mo S, et al. Liver abscess: diagnostic and management issues found in the low resource setting. Br Med Bull 2019;132(1): 45. DOI: $10.1093 \% 2 F b m b \% 2 F I d z 032$. 